\title{
A Novel Use of Liraglutide: Induction of Partial Remission in Ulcerative Colitis and Ankylosing Spondylitis
}

\section{Jeffrey Lourie*}

North Branch Health, Montpelier, Vermont, United States of America

*Corresponding author: Jeffrey Lourie, North Branch Health, Montpelier, Vermont, United States of America, Tel: +1-802760-6288, Fax: +1-802-760-6286

\begin{abstract}
Ulcerative colitis and ankylosing spondylitis treatment options are limited to a few classes of medications at this time. Those options provide improvement for some patients, but leaves many with ongoing, unremitting symptoms. We report a case of ulcerative colitis (UC) with comorbid ankylosing spondylitis (AS), in which administration of a daily subcutaneous liraglutide, an exogenous Glucagon-like Peptide-1 receptor agonist (GLP-1RA), led to symptomatic remission of UC and significant improvement in AS symptoms.
\end{abstract}

\section{Keywords}

Ankylosing spondylitis, Ulcerative colitis, GLP-1, Liraglutide

\section{Introduction}

We present a case of symptomatic remission of UC and significant symptomatic improvement in AS after initiation of daily subcutaneous liraglutide.

GLP-1 receptors (GLP-1R) are involved multiple areas of biological activity, primarily involving the GI tract and regulation of blood glucose levels. Endogenous Glucagon-like Peptide-1 (GLP-1) is a rapidly degrading incretin, facilitating glucose-dependent insulin secretion while inhibiting glucagon secretion and gastric emptying. Levels rise quickly after eating, then drop quickly. Most GLP-1 producing cells are found in the ileum and colon, providing a feedback loop between the GLP-1 receptors in the upper $\mathrm{GI}$ tract and controlling gastric motility [1]. GLP-1 is also produced in the hind-brain where it may help to regulate hunger through central nervous system activation of GLP-1R [2].

Exogenous GLP-1RA have the primary benefit of a significantly longer half-life than GLP-1 itself. Currently there are GLP-1RA molecules available which require dosing as infrequently as once a week. These exogenous GLP-1RA molecules have all demonstrated similar function to endogenous GLP-1 [3].

Previous literature has shown the likely mechanism and protective effect of GLP-1 on gastric mucosa in animal models. There are several reports indicating that GLP-1 has a role in intestinal mucosal growth [4]. Koehler, et al. demonstrated that GLP-1R signaling effects mucosal expansion of the small bowel and colon [5].

We are unable to find reports of GLP-1RA molecules leading to improvement of inflammatory bowel disease or inflammatory arthritis in humans. It is our hope that this case report will spur further research into the safety and efficacy of GLP-1RA for treatment of UC and AS in humans.

\section{Case Description}

A 42-year-old male, diagnosed with UC by colonoscopy approximately 14 years previously, with a reported account consistent with a 25 year history of active disease, and a similar history of AS diagnosed radiographically and serologically.

Treatment history for UC and AS started with oral sulfasalazine (ineffective), followed by TNF- $\alpha$ inhibition (starting with intravenous infliximab for $\sim 2$ years, then subcutaneous golimumab for $\sim 5$ years, and finally subcutaneous certolizumab pegol bi-weekly for 4 years. During the course of TNF- $\alpha$ inhibition he was also treated with methotrexate (initially $7.5 \mathrm{mg}$ weekly, ultimately titrated to $20 \mathrm{mg}$ weekly), but reports inconsistency

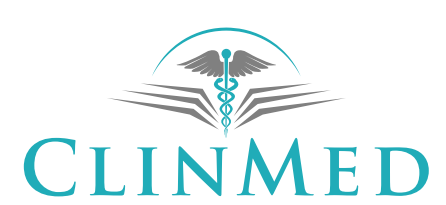

INTERNATIONAL LIBRARY 
with methotrexate use. He has also used a variety of NSAIDs on a PRN basis, which continued through the time encompassed by this report.

Subcutaneous liraglutide was initiated and slowly titrated for treatment of obesity. He made no dietary changes (other than the addition of fiber) nor modified activities or lifestyle over the course of this treatment. We were able to obtain extensive history including recent disease activity scoring.

His pre-treatment Mayo Score of Ulcerative Colitis Activity (MSUCA) was 5, and his Bath Ankylosing Spondylitis Disease Activity Index (BASDAI) score was 6.7. We have pre-treatment colonoscopy data confirming active disease, but no post-treatment data.

He reported significant improvement after 3 days of subcutaneous liraglutide $0.6 \mathrm{mg}$, and once titrated to $1.8 \mathrm{mg}$ daily reported full remission. After 30 days he had titrated to $3.0 \mathrm{mg}$ and continued to report full remission of his colitis symptoms.

Post-treatment MSUCA was decreased from 5 to 2 (within the limitations of this report, the pre-treatment colonoscopy data was used for calculation of MSUCA), and BASDAl score was decreased from 6.7 to 5.3. BMI after 30 days had decreased from 31 to 29.

Globally, symptoms were improved with the exception of fatigue. He reported remission of UC to the point of mild constipation, which he states has never been an issue for him in the past. This was relieved with the addition of a regimen of oral fiber supplementation and increased hydration. Otherwise he reported no side effects or issues with the medication (except for early-treatment mild nausea, which subsided within 3-4 days of each dose increase).

\section{Discussion}

GLP-1R signaling in the intestine and subsequent up-regulation of intestinal epithelial lymphocytes has been established as part of the inflammatory response pathway [6,7], and enteroendocrine $L$ cells increase their secretion of GLP-1 in response to systemic inflammation [8].

We speculate that the effect of liraglutide is analogous to exendin-4 decreasing mRNA expression of IL17, IL-1 $\beta$, IL- 6 and TNF- $\alpha$ as demonstrated by Lee, et al. [9] AS and UC treatment has largely been focused on TNF- $\alpha$ inhibition in the past, but AS in particular is strongly correlated with IL-6 and IL-17 as well [10]. Drucker and Rosen also noted improvement of psoriatic cutaneous manifestations with liraglutide, which may be mediated through the same channels [11].

An additional benefit may be related to increased immunocompetence in the gut. Rosario and D'Alessio reported, using a murine model, that treatment with the GLP-1RA exendin-4 resulted in "rapid, transient upregulation of antimicrobial and immunomodulatory genes in wild-type mice and reduced edema with a more normal gene expression pattern in mice with colitis" [12].

This supports a cascade of events initiated by GLP1RA, ultimately providing multifactorial (involving both intrinsic healing as well as supporting a healthy microbiome [12]) mucosal protection and healing.

\section{Conclusions}

Based on this report, more research into the efficacy and safety of exogenous GLP-1RA molecules for treatment of inflammatory bowel disease and inflammatory arthritis resistant to current treatment modalities is warranted.

This supports the idea of a broader relationship between GLP-1 and an anti-inflammatory cascade outside of the gastrointestinal tract, and previous research has already shown a positive effect on encephalomyelitis [9] as well as cutaneous psoriasis [11].

There are safety considerations which may limit immediate utility. GLP-1RA are contraindicated for patients with significant GERD or gastroparesis. Reported adverse effects of GLP-1RA medications are primarily GI, with the longer-acting agents reporting a decreased rate of adverse effects. A rare adverse event is pancreatitis (likely from rapid beta cell proliferation).

As UC predisposes for colon cancer, we are presented with some unknown risks related to neoplasia. Koehler, et al. demonstrated, in a murine model, what appears to be gene-linked biphasic modulation of intestinal neoplastic polyp growth with exendin-4, so further research is needed to ensure safety [5]. Finally, thyroid medullary carcinoma is listed as a GLP-1RA concern because it was seen in murine models, but has not been reproduced in retrospective analyses [13].

\section{References}

1. Sun EW, Fontgalland $D$ de, Rabbitt $P$, Hollington $P$, Sposato L, et al. (2017) Mechanisms controlling Glucose-Induced GLP-1 secretion in human small intestine. Diabetes 66: 2144-2149.

2. Alhadeff AL, Rupprecht LE, Hayes MR (2012) GLP-1 Neurons in the nucleus of the solitary tract project directly to the ventral tegmental area and nucleus accumbens to control for food intake. Endocrinology 153: 647-658.

3. Hinnen D (2017) Glucagon-Like Peptide 1 receptor agonists for Type 2 diabetes. Diabetes Spectrum 30: 202-210.

4. Simonsen L, Pilgaard S, Orskov C, Rosenkilde MM, Hartmann B, et al. (2007) Exendin-4 but not dipeptidyl peptidase IV inhibition, increases small intestinal mass in GK rats. American Journal of Physiology-Gastrointestinal and Liver Physiology 293: G288-G295.

5. Koehler JA, Baggio LL, Yusta B, Longuet C, Rowland KJ, et al. (2015) GLP-1R agonists promote normal and neoplastic intestinal growth through mechanisms requiring Fgf7. Cell Metab 21: 379-391.

6. Ellingsgaard $H$, Hauselmann I, Schuler B, Habib AM, Baggio LL, et al. (2011) Interleukin-6 enhances insulin 
secretion by increasing glucagon-like peptide-1 secretion from L cells and alpha cells. Nat Med 17: 1481-1489.

7. Kahles F, Meyer C, Mollmann J, Diebold S, Findeisen $\mathrm{HM}$, et al. (2014) GLP-1 secretion is increased by inflammatory stimuli in an IL-6-dependent manner leading to hyperinsulinemia and blood glucose lowering. Diabetes 63: 3221-3229.

8. Nguyen AT, Mandard S, Dray C, Deckert V, Valet P, et al. (2013) Lipopolysaccharides-Mediated increase in glucosestimulated insulin secretion: Involvement of the GLP-1 pathway. Diabetes 63: 471-482.

9. Lee C-H, Jeon SJ, Cho KS, Moon E, Sapkota A, et al. (2017) Activation of glucagon-like peptide-1 receptor promotes neuroprotection in experimental autoimmune encephalomyelitis by reducing neuroinflammatory responses. Mol Neurobiol 55: 3007-3020.
10. Lin T-tian, Lu J, Qi C-yue, Yuan L, Li X-lin, et al. (2014) Elevated serum level of IL-27 and VEGF in patients with ankylosing spondylitis and associate with disease activity. Clin Exp Med 15: 227-231.

11. Drucker DJ, Rosen CF (2011) Glucagon-like peptide-1 (GLP-1) receptor agonists obesity and psoriasis: Diabetes meets dermatology. Diabetologia 54: 2741-2744.

12. Rosario W, D'Alessio D (2015) An innate disposition for a healthier gut: GLP-1R signaling in intestinal epithelial lymphocytes. Diabetes 64: 2329-2331.

13. Liang C, Bertoia ML, Ding Y, Clifford CR, Qiao Q, et al. (2018) Exenatide use and incidence of pancreatic and thyroid cancer: A retrospective cohort study. Diabetes Obesity and Metabolism 21: 1037-1042. 